\title{
Animated Transitions for Adaptive Small Size Mobile Menus
}

\author{
Jussi Huhtala $^{1}$, Jani Mäntyjärvi ${ }^{1,2}$, Aino Ahtinen ${ }^{1}$, Leena Ventä ${ }^{1}$, \\ and Minna Isomursu ${ }^{2}$ \\ ${ }^{1}$ Nokia Research Center, P.O. Box 300, FI-90230, OULU, Finland \\ ${ }^{2}$ VTT Technical Research Centre of Finland, P.O. Box 1100, FI-90571, OULU, Finland \\ \{jussi.huhtala, aino.ahtinen, leena.venta\} @nokia.com, \\ \{jani.mantyjarvi, minna.isomursu\} @vtt.fi
}

\begin{abstract}
This paper explores how the user interface of a mobile device could support human perception and conception of changes in its environment. Animated transition effects may hold potential for visualizing changes in the resources available for the user through the context-aware user interface. Four different transition types are investigated. Each transition represents a different approach for visualizing changes in UI. The transitions are evaluated with 40 test subjects, half in India and half in Europe. Statistical analysis of the results indicates that animated transition effects have a clear positive effect on perception and conception of change.
\end{abstract}

Keywords: Mobile UI design, animation, transition.

\section{Introduction}

In ubiquitous computing, all computing devices are aware of their surrounding devices and their available resources. A mobile device could potentially act as a generic device for providing user interfaces (UIs) for a smart environment. When a user moves from a smart space to another, digital affordance available for the user through a mobile UI changes, too. This may set challenges for a user since (s)he has gotten accustomed to the UI structure of his/her device.

This paper explores how the user interface of the mobile device could visually support the user to perceive and comprehend the changes in the resources available. The purpose of this research is to study user perception of dynamic grid menus which have changing elements depending on the space a user experiences at the moment. This study assumes that there is technology available, which provides information on the new smart spaces and all available resources of a given place to a mobile device before the user arrives to this place [1].

Research on perception and motion on a screen is quite novel in the mobile UI domain. However, considerable research work has been carried out on human visual perception in the realm of desktop UI. Animations have been reported to improve user performance and user experience. In the research by Bladh et al. it has been found that short animations during navigation with 3D maps affect user performance and reduce task times when e.g. counting details on maps [2]. Klein \& Bederson measured better 
results in reading when scrolling animation was used [3]. The results of Baecker et al. show that small animated icons can convey functionality better than static icons of the same size. [4]. Baudish et al. present how animated effects have been used successfully to improve the user's ability to perceive changes on a computer desktop [5]. With careful design, the transitions can be designed and implemented so that they do not cause delays or disturb the task performance. Study by Hong et al. shows that flashing animation apparently helps users to locate single targets within a larger amount of information [6]. However, flashing has also been found to disturb the user if the information density is not high or flashing does not happen in a targeted item. Several studies concerning failures of visual awareness show that pictures separated by a blank frame cause a change blindness effect [7]. These results can help to understand visual perception in computer graphics and set a baseline for animation perception.

Studies on the use of animated transitions have usually been done in desktop environments. In related work, moving screens or elements are used to help with navigation and draw the attention of the user to selected user interface elements. The work presented here explores how animations could make the user more aware of the changes in the digital resources available in varying environments.

This paper is organized as follows: Animated transitions are explained in the next section, after which the experiments are described. Then, results are provided, and finally, concluding remarks are presented.

\section{Animated Transitions}

We used a demonstration application that visualized a hypothetical situation: A user moving from one space to another and a UI presenting the new resources available. The application presented twelve icons arranged in a grid type menu. The icons were selected to represent different digital resources. We used Crystal Project [8] icons designed for open source environments because they were not widely used in mobile devices. Some of them were widely known figures like the RSS feed icon and others represented general features like device icons (Figure 1). The selected 48 icons had
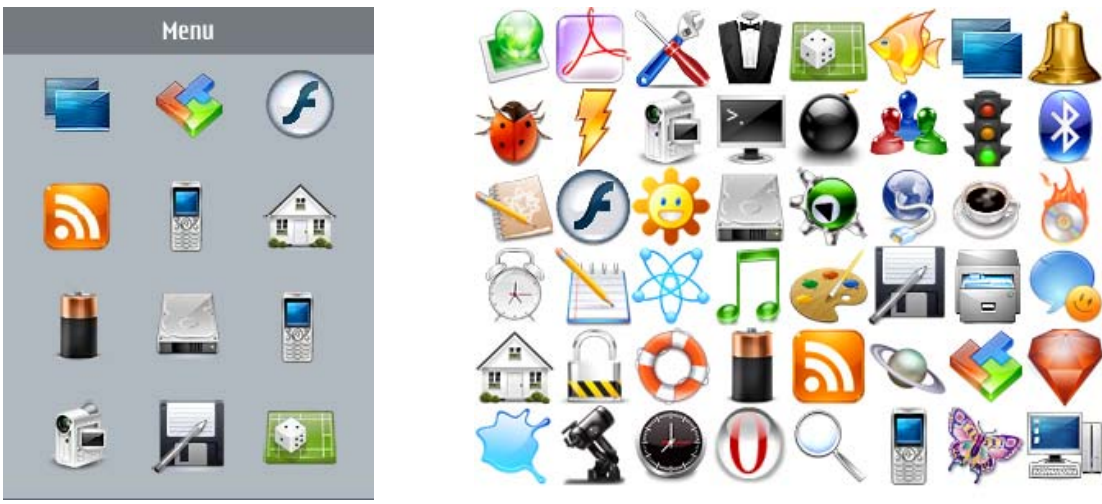

Fig. 1. Mobile grid menu and all icons used in the study 
various overall colorings and outline shapes. Text was not used because we wanted to keep the visual information clear and reduce the effect of different linguistic backgrounds.

Four different transition types were chosen as options for presenting change. The first two transition types were not animated, and the last two represented different, both common, animated transition types. The transition types with their implementation are described in detail in the following.

Blank screen transition (change blindness effect) displays a grey screen for the duration of 1,2, or 3 seconds between the screens. Variations of blank screen transitions are common, for example, in applications where a box with text "Updating..." is displayed on the screen while updating is in progress. Even partial covering of the screen can cause change blindness which makes it difficult to notice the change.

Direct change transition switches the icons immediately without any animation. Direct change does not give any visual cue that a change is about to happen.

Sliding animation accelerates the disappearing icon to the bottom right, representing the resource becoming unavailable. The new icon appears simultaneously by sliding into the menu from the upper left corner of the screen. The new icon slows its speed before placing itself in the grid to emphasize grounding and staying in place. The sliding animation is illustrated in Figure 2.

Blinking animation first pulses the icon representing the old resource to partial transparency two to six times, depending on the duration of transition, after which it disappears from the screen. After that, the icon representing the new available resource appears first as a white silhouette of the icon and then smoothly tones down to its normal coloring. The blinking effect is illustrated in Figure 3.

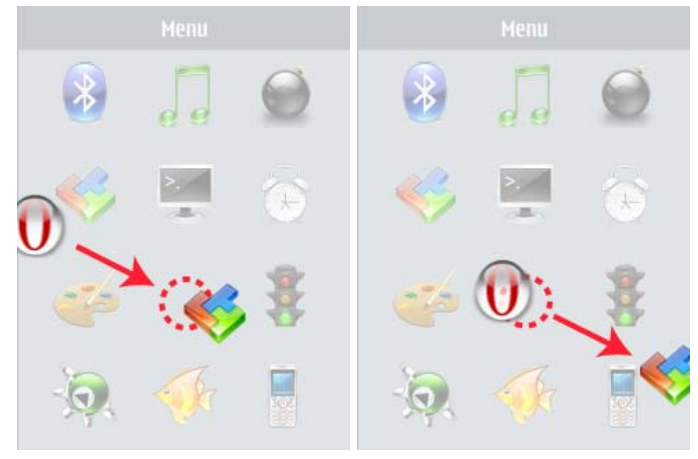

Fig. 2. Sliding animation

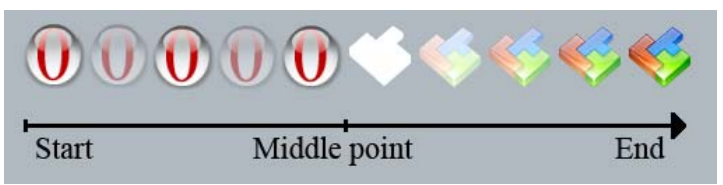

Fig. 3. Blinking animation 


\section{Experiment}

The transition effects were explored from the viewpoints of user performance and subjective satisfaction.

The experiment was conducted with 40 voluntary participants. 20 of them were from Finland and 20 from India. The test subjects were between the ages of 20 and 40 years (average about 30 years). Most participants used mobile phones with graphical user interfaces regularly, and had a technical or office work background. 16 of the test subjects were female and 24 were male.

\subsection{Experiment Settings}

The demonstration application ran on the Nokia N95 mobile phone and the Flash Lite application. The test was performed indoors in a place where the illumination conditions were even, no distractions were present, and the users were able to physically move from one space to another. Data collection was done by a researcher who shadowed the subjects during the experiment. The experiment procedure was as follows:

The user was given a mobile device and a pen. The user held the pen in the hand they used for writing, and the mobile phone in the other hand. The following task was repeated 18 times by each user.

1. The user was requested to walk a couple of meters, and open the blank screen by pressing "Enter."

2. When the application started, the user first saw a grid menu with the resources of the "old location" on the mobile device screen for the duration of five seconds.

3. Then one of the four transitions was used to change two, four or six icons in different locations of the screen simultaneously. The transition times varied between one and three seconds.

4. The new combination of resource icons was displayed to the user for the duration of five seconds, after which the screen turned black (see timeline in Figure 4).

5. The researcher presented the data collection form to the user, who then used it to report the changes he or she had been able to perceive. The data collection form followed the structure presented in Figure 5.

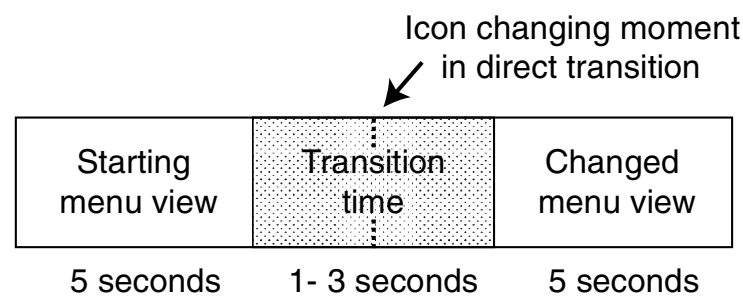

Fig. 4. Timeline of the application

As we used four different transition types, three different transition durations, and three different amounts of changing icons, there was a total of 36 different combinations to test. To maintain the participant's attention, each user tested only 18 of all the 
combinations. The application automatically randomized the icon graphics to make the icon grid look different each time. In addition, we allowed multiple similar icons on the screen because this is possible in real-world situations as well. The new icons after transition were always different from the ones they replaced.

The locations of the changing icons were randomized beforehand in three different sets of tasks to reduce the effect of easily recognized patterns. The distribution between sets was 7-6-7.

The data collection form shown in Figure 5 was presented to the test subject. Places where the user had perceived change were marked on the answer cells representing the grid menu. To prevent a learning effect, the transitions were not presented to the user beforehand. The order of tasks was also varied between users.

After the tasks, users gave subjective ratings from 1 (low) to 5 (high) to rank the visual appearance, clarity, and usefulness of the transition types. Also, subjective comments on the complexity of the task and general remarks were requested.

\begin{tabular}{|c|c|c|c|c|c|}
\hline \multicolumn{6}{|c|}{ Rate the visual appearance } \\
\hline & \multicolumn{2}{|l|}{$\begin{array}{l}\text { Not visualliy } \\
\text { appealing }\end{array}$} & \multicolumn{2}{|l|}{ Can't say } & $\begin{array}{c}\text { Visually } \\
\text { appealing }\end{array}$ \\
\hline Direct transition & 1 & 2 & 3 & 4 & 5 \\
\hline Blank screen & 1 & 2 & 3 & 4 & 5 \\
\hline Moving icons & 1 & 2 & 3 & 4 & 5 \\
\hline Blinking icons & 1 & 2 & 3 & 4 & 5 \\
\hline \multicolumn{6}{|c|}{ Rate the clearness } \\
\hline & \multicolumn{2}{|l|}{ Unclear } & \multicolumn{2}{|l|}{ Can't say } & Very clear \\
\hline Direct transition & 1 & 2 & 3 & 4 & 5 \\
\hline Blank screen & 1 & 2 & 3 & 4 & 5 \\
\hline Moving icons & 1 & 2 & 3 & 4 & 5 \\
\hline Blinking icons & 1 & 2 & 3 & 4 & 5 \\
\hline \multicolumn{6}{|c|}{ Rate the usefulness } \\
\hline & \multicolumn{2}{|l|}{ Not useful } & Can't say & \multicolumn{2}{|r|}{ Very useful } \\
\hline Direct transition & 1 & 2 & 3 & 4 & 5 \\
\hline Blank screen & 1 & 2 & 3 & 4 & 5 \\
\hline Moving icons & 1 & 2 & 3 & 4 & 5 \\
\hline Blinking icons & 1 & 2 & 3 & 4 & 5 \\
\hline
\end{tabular}

Fig. 5. Part of answer sheet, and ratings sections in end question sheet

\subsection{Analysis of Data}

The data collection forms were analyzed through the procedure illustrated in Figure 6.

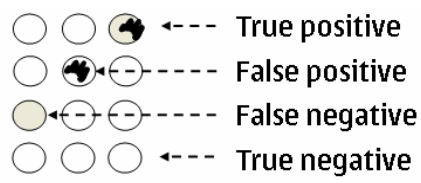

Fig. 6. Answer sheet shell and all four possible answers. Shaded circles are the locations where icons actually changed. 
Precision and recall are commonly used to measure performance. Precision is the number of correctly marked locations divided by the number of all marked locations, and recall is the number of correctly marked locations divided by the total number of all actually changed icons.

$$
\operatorname{Pr} \text { ecision }=\frac{t p}{t p+f p}, \operatorname{Re} \text { call }=\frac{t p}{t p+f n}
$$

We used Rijsbergen's F-measure [9] value to compare the different task groups. The harmonic mean of the Precision and the Recall values gave the probability of getting the correct answers from users. Then the F-measure values of different sets (transition types, durations, and amounts of icons) were compared.

$$
F=2 \cdot \frac{\operatorname{Pr} \text { ecision } \cdot \operatorname{Re} \text { call }}{\operatorname{Pr} \text { ecision }+\operatorname{Re} \text { call }}
$$

The subjective ratings consisted of numerical ratings and free-form verbal comments. Mean values were counted from numerical ratings, as variation was very small and preferences consistent between users. Verbal comments were grouped into themes, and they were used to find explaining factors for the quantitative results.

\section{Results}

Here, we first discuss the results from performance analysis and move then to the analysis of subjective ratings.

\subsection{Performance Evaluation}

\section{Effect of transition type}

The F-measure values were analyzed by using one-way ANOVA tests using transition type (four groups), duration (three groups), and icon amount (three groups) as independent variables. After that, Tukey's post-hoc test was applied to compare significant groups.

Table 1. Sums of true positives, false positives, false negatives and true negatives

\begin{tabular}{lcccc} 
Transition & tp & fp & fn & tn \\
\hline Direct & 450 & 50 & 270 & 1390 \\
Empty & 159 & 160 & 561 & 1280 \\
Blinking & 666 & 21 & 54 & 1419 \\
Sliding & 642 & 39 & 78 & 1401
\end{tabular}

Animated transitions proved to be significantly more effective at supporting the subjects in perceiving the change in the resources (Figure 7). In multiple comparisons, no statistical difference was found between the animated transitions (blinking versus 


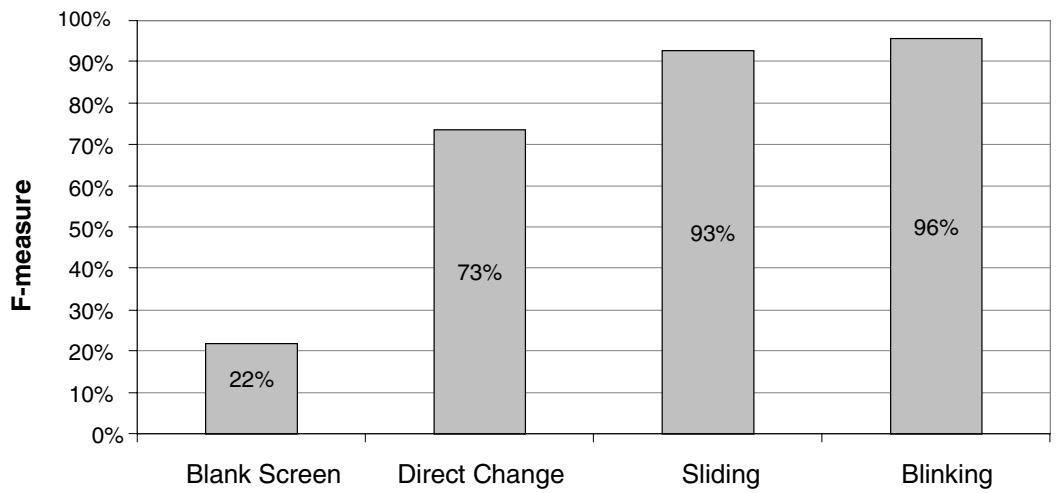

Fig. 7. Effect of transition types

sliding). However, comparisons of all other transition pairs were significant $(\mathrm{p}<.05)$. Results show that the chance of getting a right answer with the blank screen transition was remarkably low. Performance with direct transitions was significantly better, but still significantly poorer than with animated transitions.

\section{Duration and amount}

The ANOVA test did not show significant differences between duration groups or between amount groups.

Varying the duration of animations between one, two and three seconds did not have a significant impact on the results $(\mathrm{F}(2,717)=0.518, \mathrm{p}>.05)$. One-second animations worked as well as three-second ones. This is in agreement with the menu transitions study of Bladh et al [2].

Varying the amount of simultaneously changing icons also had no statistically significant impact $(\mathrm{F}(2,717)=2.41, \mathrm{p}>.05)$. However, when comparing icon amounts and transition types together, there seemed to be a small tendency that blank screen was better with a larger amount of icons (Figure 8).

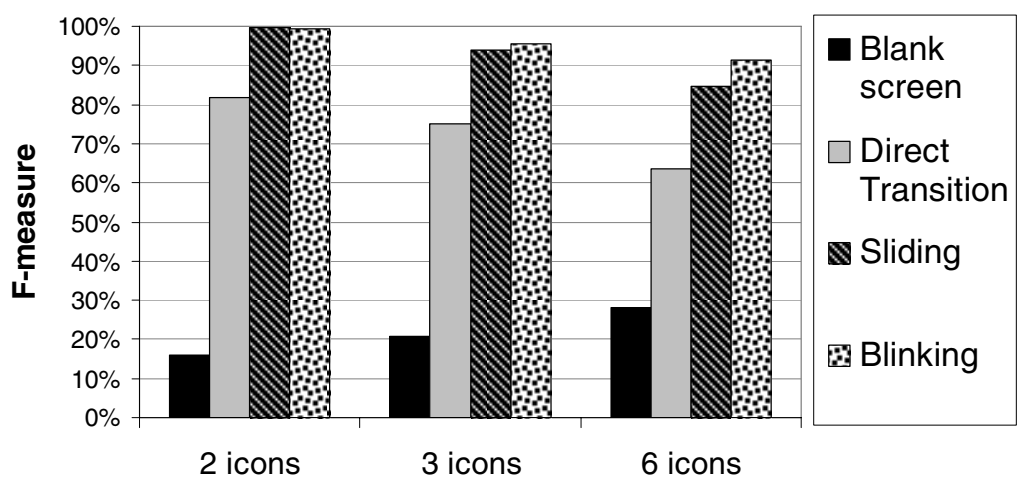

Fig. 8. Effect of icon amount with different transitions 


\subsection{Subjective Ratings}

Our analysis shows that the subjective ratings of the test subjects follow the findings of the performance evaluation. In addition, there was little variation in ratings. The transitions resulting in the best user performance were also rated most visually appealing, clear and useful. Also, there was not much variation in individual ratings between visual appearance, clarity, and usefulness. This might indicate that the test subjects were not able to express their experience through dividing it into the three components presented.

Users commented that a blank screen between two screens required more active memorizing and resulted in more cognitive load than the other transition types. With blank screen transitions, the users sometimes stated that they did not see anything changing, or that everything changed. This might explain high error rates. Change blindness forced users to make assumptions and guesses, and perhaps also to use special memorizing techniques (Figure 9).

Opinions of direct transition were more neutral. Test subjects stated that several icons changing simultaneously was confusing, and they thought they blinked just when change occurred. It is possible that this situation can be compared to change blindness. With animated transitions, this kind of problem was not mentioned (Figure 10).

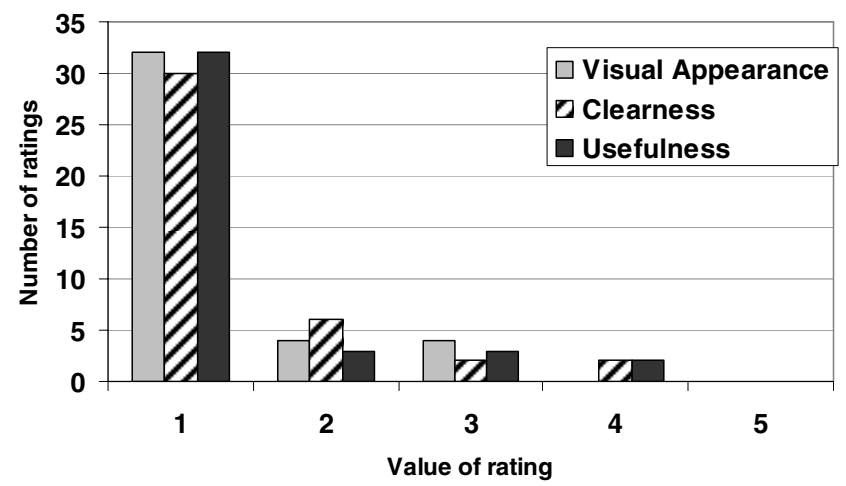

Fig. 9. Opinions of blank screen

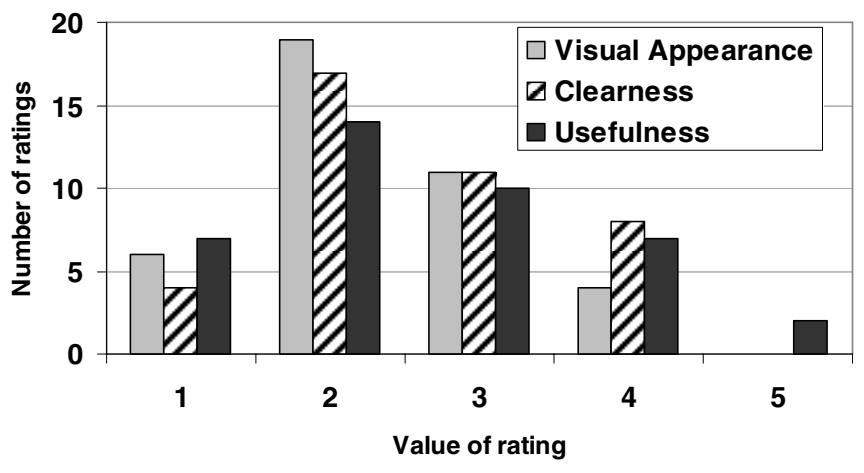

Fig. 10. Opinions of direct change 
Blinking animation was the most liked, but no significant difference was noted from sliding animation (Figure 12). Blinking was mentioned as being enjoyable and clear, but also slightly annoying. Some stated that the white color effect could last longer (Figure 11).

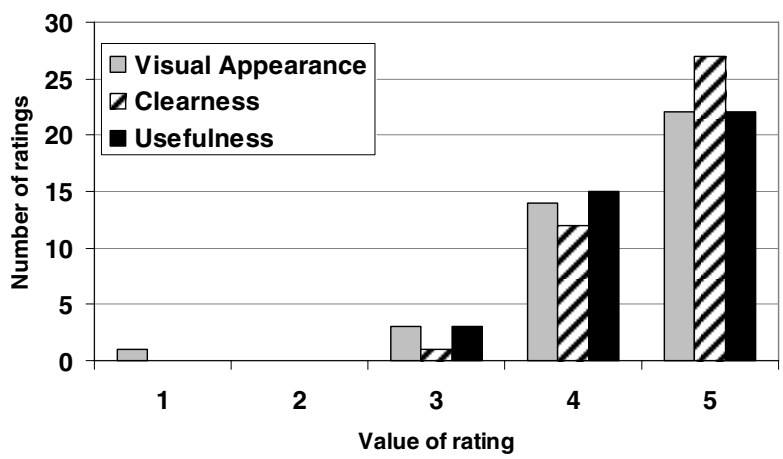

Fig. 11. Opinions of blinking animation

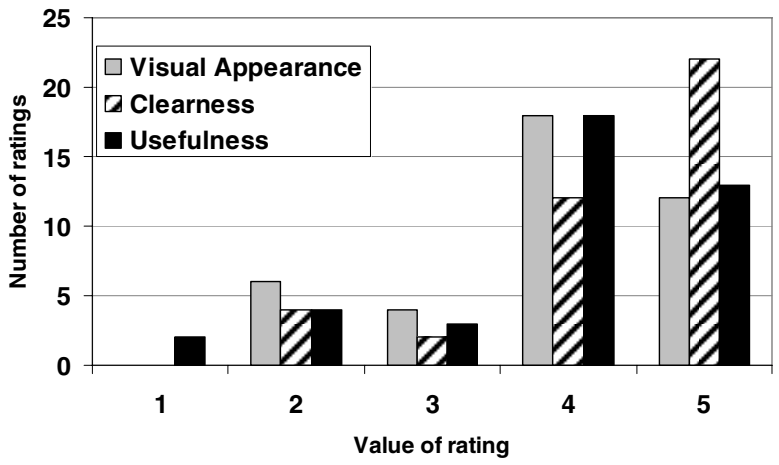

Fig. 12. Opinions of sliding animation

Sliding was liked, too, but multiple icons sliding on the screen simultaneously was perceived as confusing. Overlapping of icons was also perceived as disturbing. Sliding icons caught users' attention in a positive way. There were some slight differences in subjective ratings between blinking and sliding animations. Users rated visual appearance and usefulness a bit poorer for the sliding transition. However, this difference was not clearly supported by user comments. The most interesting detail was that the sliding animation was hoped to last for a shorter time, but the blinking one was hoped to last longer.

Users wished that the dynamic and static parts of menus should be marked clearly in the user interface. Some users mentioned that the icons should look different enough that they are not confused accidentally. 


\section{Concluding Remarks}

The animated transitions resulted in significantly better user performance compared with non-animated transitions. Even though transition time did not have an effect on user performance, subjective experiences revealed interesting issues about timing. Users hoped that sliding animations would be faster, but blinking was hoped to last longer. Experimenting with more variation in animation timing could be a subject of further research.

Blinking was perceived as a little annoying, but it was seen as a clear indicator. Sliding icons were perceived as confusing when they overlapped other icons. More detailed comparison between moving and blinking animations would offer more information how to use the benefits of animation on small screens. It appears that human visual perception can accurately handle quite complex movement on the screen. The challenge in design is to attract the user's attention without losing the appeal of the user interface. The role of animation design, acceleration in animation, and color effects with blinking animation are interesting issues for future work.

Even though the test was performed in a mobile setting simulating real-life usage situations, the users seemed to have problems in understanding the concept of changing resources and adaptive UI. A truer level of experimental realism would be needed to evaluate issues beyond perception and comprehension, such as usability and utility.

\section{References}

1. Such an enabling technology could be e.g. NoTA, http: / / www . notaworld.org

2. Bladh, T., Carr, D.A., Kljun, M.: The Effect of Animated Transitions on User Navigation in 3D Tree-Maps. In: Proceedings of the Ninth International Confer-ence on Information Visualisation (IV 2005), pp. 297-305. IEEE Computer Society, Los Alamitos (2005)

3. Klein, C., Bederson, B.B.: Benefits of animated scrolling. In: Conference on Human Factors in Computing Systems (CHI 2005), pp. 1965-1968. ACM Press, New York (2005)

4. Baecker, R., Small, I., Mander, R.: Bringing icons to life. In: Conference on Human Factors in Computing Systems (CHI 1991), pp. 1-6. ACM Press, New York (1991)

5. Baudisch, P., Tan, D., Collomb, M., Robbins, D., Hinckley, K., Agrawala, M., Zhao, S., Ramos, G.: Phosphor: Explaining Transitions in the User Interface Using Afterglow Effects. In: Proceedings of UIST 2006, pp. 169-178. ACM Press, New York (2006)

6. Hong, W., Thong, J.Y.L., Tam, K.-Y.: Does Animation Attract Online Users' At-tention? The Effects of Flash on Information Search Performance and Perceptions. Information Systems Research. INFORMS 15(1), 60-86 (2004)

7. Mancero, G., Wong, W., Amaldi, P.: Looking but not seeing: implications for HCI. In: Proceedings of ECCE 2007, pp. 167-174. ACM, New York (2007)

8. The Crystal Project is released under GNU General Public License, http: //www. everaldo.com/crystal/

9. Van Rijsbergen, C.J.: Information Retrieval. Butterworth-Heinemann, Newton (1979) 